\title{
Obturator hernia of Richter type: a diagnostic dilemma
}

\author{
Cathal Hayes (ㅇ), Karl Schmidt, Yuwaraja Neduchelyn, Ivan Ivanovski
}

General Surgery, Wexford General Hospital, Wexford, Ireland

\section{Correspondence to \\ Cathal Hayes; \\ cathalhayes@rcsi.ie}

Accepted 6 December 2020

Check for updates

(c) BMJ Publishing Group Limited 2020. No commercial re-use. See rights and permissions. Published by BMJ.

To cite: Hayes C, Schmidt K, Neduchelyn $\mathrm{Y}$, et al. BMJ

Case Rep 2020;13:e238252.

doi:10.1136/bcr-2020-

238252

\section{SUMMARY}

An 85-year-old malnourished woman presented with symptoms of small bowel obstruction of uncertain aetiology. She had presented numerous times over the previous 2 years with symptoms of left groin and thigh pain, vomiting and abdominal distension. A CT of her abdomen and pelvis ultimately revealed a leftsided pelvic hernia, between the obturator internus and pectineal muscles. This was consistent with an obturator hernia. Diagnostic laparoscopy confirmed an obturator hernia of Richter type, incarcerated within the left obturator canal. Reduction revealed a hernia sac containing viable small bowel. A primary repair was performed using a double-layer suture technique to both close and plug the hernia defect. The patient rapidly recovered following hernia repair, with resolution of all previous long-standing symptoms. This case exemplifies the typical presentation of an obturator hernia and the diagnostic challenge it poses to clinicians.

\section{BACKGROUND}

An obturator hernia describes the protrusion of abdominal viscera through the obturator foramen. It is a relatively rare type of abdominopelvic hernia, accounting for only $0.05 \%-1.4 \%$ of all hernias. ${ }^{1}$

The obturator foramen is an oval-shaped opening located at the anterolateral aspect of the pelvic wall, formed by the ischial and pubic rami. It is covered by a fibrous obturator membrane, within which a natural defect exists (obturator canal) to allow passage of the obturator nerve, artery and vein from the pelvis to the medial thigh.

The obturator canal is filled with preperitoneal fat and lymphatic tissue, which provide a supportive cushion for the neurovascular bundle which traverses it. Obturator membrane laxity, coupled with loss of this tissue cushion, results in relative widening of the obturator canal, allowing for passage of abdominal contents through this.

Obturator hernias occur predominantly in small, elderly, multiparous women with a history of weight loss. Multiple pregnancies within a narrow pelvis results in ligamentous laxity and weakening of the obturator membrane. This coupled with loss of fatty cushioning in the context of cachexia, predisposes these patients to a widened obturator canal and an increased risk of obturator hernia development. It is aptly nicknamed the 'little old woman's hernia'. ${ }^{2}$

Its presentation is varied, but can present with incarceration and acute intestinal obstruction. Its detection can be challenging and often delayed due to the lack of specific signs which characterise other incarcerated hernias. This delay in diagnosis and treatment can trigger irreversible injury to the hernia contents, resulting in gangrenous bowel, viscus perforation, peritonitis and eventually death. Mortality rates vary, with estimates ranging from $12 \%$ to $70 \% .^{3}$

Clinical examination is often unremarkable. A proximal thigh lump may be felt between the pectineus and adductor muscles. Medial thigh ecchymosis may be evident in the context of bowel necrosis. The Howship-Romberg sign is characterised by pain extending from the inguinal crease, along the medial thigh down to level of the knee. Worsened by coughing and thigh adduction, it represents compression of the cutaneous branch of the obturator nerve. ${ }^{3}$

Given the limitations of clinical examination, other diagnostic modalities are often required. Plain film radiography may identify signs of bowel obstruction and rarely an air-fluid level in the area of the obturator foramen; however, this imaging modality is rarely useful in diagnosis. ${ }^{4}$ Ultrasonography remains a cheap, non-invasive alternative, which may identify a hypoechoic mass posterior to the pectineus. However, this would only be identified by a skilled technician alerted to a possible obturator hernia specifically scanning the femoral region. ${ }^{5}$ CT remains the gold standard for detection, boosting preoperative diagnosis rates to $90 \% .^{3}$

Definitive treatment involves reduction and repair of the hernia defect. Numerous approaches have been described including open transabdominal, inguinal, obturator and more recently laparoscopic (either extraperitoneal or transperitoneal) approaches. Defect closure can involve primary suture closure in the context of a small defect, or plugging with native tissue such as omentum, peritoneal patch, pectineal muscle flap or even plugging with an ovary. More recently, the trend has been to repair these defects with polypropylene mesh as they are strong, non-absorbable and potentially resistant to infection. ${ }^{6}$

Our patient underwent a successful laparoscopic transperitoneal approach, with instrumental reduction of hernia contents to reveal a $1 \mathrm{~cm}$ hernial defect. Primary repair was performed in preference to preperitoneal mesh repair given the patient's haemodynamic instability at the time of surgery and the potential mortality risk associated with a more time-consuming mesh repair.

\section{CASE PRESENTATION}

An 85-year-old malnourished woman presented emergently to our institute with symptoms of small bowel obstruction (SBO). Her history was 
significant for hypertension, osteoarthritis, right total hip arthroplasty, hysterectomy and nephrectomy for a benign renal lesion many years previously.

She had presented multiple times over the previous 2 years with symptoms of left-sided abdominal and groin pain associated with vomiting. A presumptive diagnosis of diverticulitis had been made in 2018, with subsequent endoscopy confirming the presence of sigmoid diverticulosis. A repeat presentation of groin and left medial thigh pain in 2019 resulted in a presumptive diagnosis of arthralgia secondary to osteoarthritis, which triggered an orthopaedic referral for hip arthroplasty.

Most recently, she presented with bilious vomiting, abdominal distension, absent bowel sounds and obstipation. She was diagnosed with an SBO and admitted for treatment and diagnostics.

\section{INVESTIGATIONS}

Laboratory investigations revealed an elevated white cell count $\left(10.5 \times 10^{9} / \mathrm{L}\right)$ and $\mathrm{C}$ reactive protein $(213)$. An acute kidney injury was also evident, with a serum urea $(21.0 \mathrm{~g} / \mathrm{dL})$ and creatinine $(132 \mathrm{~g} / \mathrm{dL})$ being elevated.

Plain film imaging of her abdomen revealed gaseous small bowel distension with a diameter up to $4.9 \mathrm{~cm}$ projected over the pelvic outlet-this was consistent with the clinical diagnosis of SBO.

Following admission, she was treated with wide-bore nasogastric (NG) drainage, fluid resuscitation, analgesia and antiemesis. She was admitted to the ward and underwent a CT of her abdomen and pelvis the following morning.

The contrast CT abdomen and pelvis reported by a consultant radiologist identified distended, fluid-filled loops of small bowel in the pelvis, with a suspected transition point in the left inguinal canal. As such, a preliminary diagnosis of SBO secondary to inguinal herniation was made. Following interdiciplinary discussion, a second hernia in the obturator canal was identified. This was ultimately the cause of her symptoms.

\section{TREATMENT}

On day 1 of admission, the patient became unwell with haemodynamic instability and a broad-complex ventricular tachycardia-she was transferred to the intensive care unit for invasive monitoring and rhythm control with intravenous amiodarone. Clinical examination did reveal an inguinal hernia (as suggested initially by CT), yet this was soft and reducible. Given the clinical picture of SBO, coupled with radiological findings, the patient was transferred to theatre where she underwent a Liechtenstein repair of an inguinal hernia.

Despite this repair, over the following days the patient's symptoms of SBO failed to resolve, with persistent large volumes of bilious fluid aspirated from her NG tube.

Following interdepartmental review of the initial CT imaging, a second hernia between the obturator internus and pectineus muscles was identified-this was consistent with an obturator hernia (figure 1).

Following extensive discussion regarding the potential morbidity and mortality risks associated with further surgical intervention, the patient was transferred to theatre for repair. A diagnostic laparoscopy confirmed the presence of an obturator hernia of Richter type incarcerated within the left obturator canal (figure 2). A loop of ileum was tightly adherent to the obturator hernia sac, reflecting the likely long-standing nature of this hernia.

Following reduction, the affected segment of ileum was noted to be healthy and a primary repair of the hernia defect was

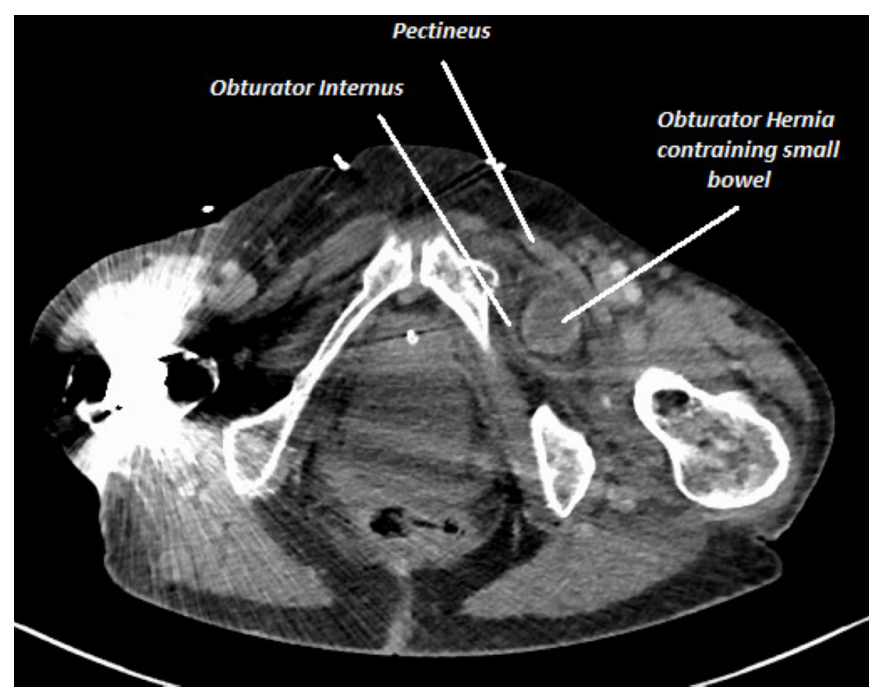

Figure 1 Axial CT pelvis demonstrating obturator hernia between pectineus and obturator internus muscles. Significant artefact from previous right hemiarthroplasty implant.

performed. Suture repair was carried out with Prolene using a double-layer technique. The first layer resulted in closure of the defect, the second layer created a peritoneal plug to fill the defect and reduce recurrence risk.

\section{OUTCOME AND FOLLOW-UP}

Following definitive repair of her obturator hernia, the patient's symptoms of SBO resolved. Ward-based multidisciplinary input from the surgical, dietetic and physiotherapy teams resulted in rapid progress.

Day 1 saw her NG tube removed. Day 2 saw her return to an oral diet. Day 3 saw her independent of any intravenous support. Day 4 saw her mobile and independent. She has since been surgically discharged and remains in the community.

\section{DISCUSSION}

This case exemplifies the diagnostic challenge associated with obturator hernias. Our patient first presented in 2018 with longstanding symptoms of abdominal, groin and medial thigh pain. A number of diagnoses were made to account for her symptoms, including diverticulitis and arthralgia secondary to osteoarthritis. In hindsight, her symptoms over this 2 -year period were consistent with a symptomatic obturator hernia. Her medial thigh pain likely reflected a positive Howship-Romberg sign and compression of the cutaneous branch of her obturator nerve.

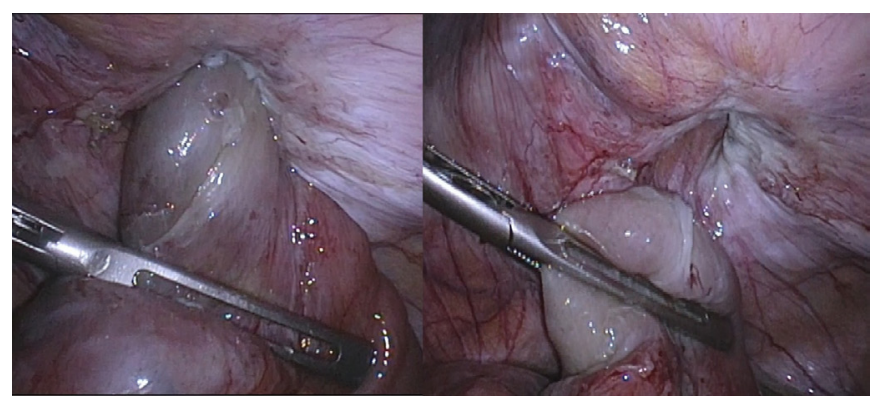

Figure 2 Obturator hernia of Richter type-prereduction and postreduction. 


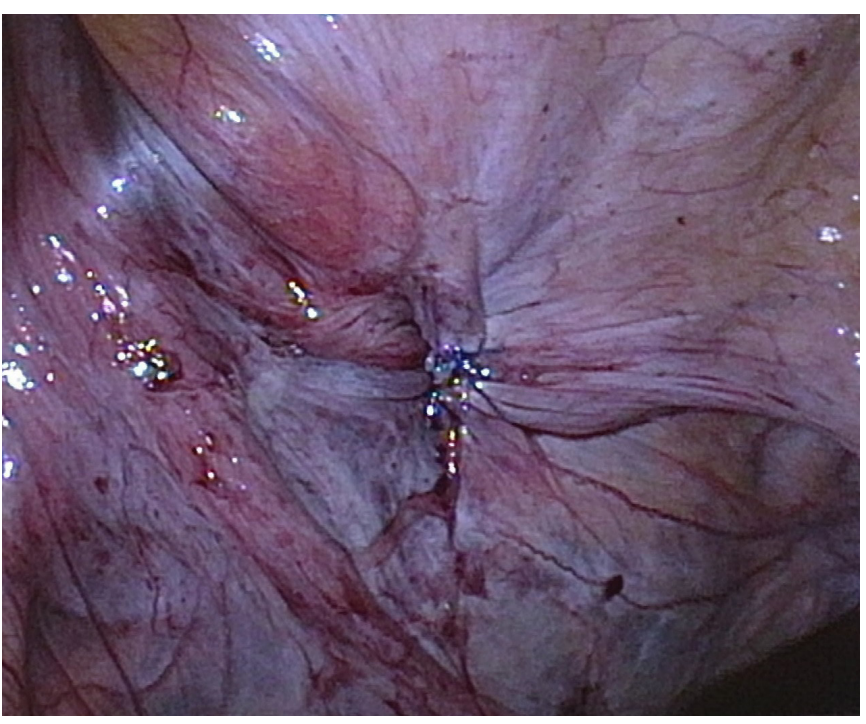

Figure 3 Post-herniorraphy with two-layer suture technique. First layer to close defect, second layer to create peritoneal plug to fill defect.

It was her presentation with SBO and haemodynamic instability which triggered an emergent admission and CT imaging. Despite acquiring these images, the underlying diagnosis of obturator hernia was still elusive. An incidental inguinal hernia was thought to be the culprit and subsequently repaired. Only after interdepartmental review was the underlying pathology fully determined. Following this, the appropriate treatment was rapidly instigated with a successful outcome.

As well as a typical clinical course, this case also exemplifies the typical profile of a patient affected by an obturator hernia. Our patient was elderly, multiparous, with a history of cachexia and weight loss. As such, she meets many of the risk factors discussed above-indeed, the 'little old female hernia' description is appropriate.

Although there exists no consensus as to optimal repair, the current trend involves the use of synthetic mesh material via laparoscopic approach (either extraperitoneal or transperitoneal), with recurrence rates as low as $10 \%$ reported. ${ }^{7}$ Mesh implantation would be contraindicated in the context of peritoneal contamination or an enteric resection. Our patient had neither of these contraindications, yet given her requirement for significant inotropic support intraoperatively, a decision was made to proceed with the least time-consuming procedure. As such, a herniorraphy using a double-layered technique with Prolene suture was carried out (figure 3). The first layer closed the hernia defect, the second layer allowed for a peritoneal plug to fill the defect. ${ }^{8}$

Mortality rates associated with obturator hernia repairs are cited as high as 70\%. ${ }^{19}$ This reflects the delay in diagnosis and resultant complications including hernia strangulation, perforation and abdominal sepsis often seen in these cases. Our patient had an obturator hernia of Richter type without these complications, affording her the chance of a successful outcome.

\section{Learning points}

- Obturator hernias often present with a protracted clinical course and non-specific symptoms. This poses a diagnostic dilemma for clinicians.

- A high index of suspicion must be maintained in elderly, cachectic, multiparous women with symptoms of small bowel obstruction.

- CT imaging of abdomen and pelvis remains the most sensitive diagnostic tool for identifying obturator hernias.

- Definitive repair with placement of preperitoneal mesh provides the most definitive solution. This is contraindicated in cases of peritoneal contamination.

- Mortality rates between $12 \%$ and $70 \%$ have been quoted reflecting the frequently delayed diagnosis and resultant complications of hernia incarceration, strangulation and perforation.

\section{Twitter Cathal Hayes@Cathalhayes6}

Contributors $\mathrm{CH}$ prepared the manuscript draft with significant intellectual input from II and YN, KS was tasked with critical revision and editorial oversight. YN obtained patient consent. All authors approved the final manuscript

Funding The authors have not declared a specific grant for this research from any funding agency in the public, commercial or not-for-profit sectors.

Competing interests None declared.

Patient consent for publication Obtained.

Provenance and peer review Not commissioned; externally peer reviewed.

\section{ORCID iD}

Cathal Hayes http://orcid.org/0000-0001-5540-9522

\section{REFERENCES}

1 Chang S-S, Shan Y-S, Lin Y-J, et al. A review of obturator hernia and a proposed algorithm for its diagnosis and treatment. World J Surg 2005;29:450-4.

2 Bjork KJ, Mucha P, Cahill DR. Obturator hernia. Surg Gynecol Obstet 1988;167:217-22.

3 Mandarry MT, Zeng S-B, Wei Z-Q, et al. Obturator hernia--a condition seldom thought of and hence seldom sought. Int J Colorectal Dis 2012;27:133-41.

4 Uludag $M$, Yetkin $G$, Kebudi A, et al. A rare cause of intestinal obstruction: incarcerated femoral hernia, strangulated obturator hernia. Hernia 2006;10:288-91.

5 Gilliam A, O'Boyle CJ, Wai D, et al. Ultrasonic diagnosis of strangulated obturator hernia. Eur J Surg 2000;166:420-1.

6 Lobo DN, Clarke DJ, Barlow AP. Obturator hernia: a new technique for repair. J R Coll Surg Edinb 1998;43:33-4.

7 Rodríguez-Hermosa JI, Codina-Cazador A, Maroto-Genover A, et al. Obturator hernia: clinical analysis of 16 cases and algorithm for its diagnosis and treatment. Hernia 2008;12:289-97.

8 Martínez Insua C, Costa Pereira JM, Cardoso de Oliveira M. Obturator hernia: the plug technique. Hernia 2001;5:161-3.

9 Yokoyama Y, Yamaguchi A, Isogai M, et al. Thirty-Six cases of obturator hernia: does computed tomography contribute to postoperative outcome? World J Surg $1999 ; 23: 214-7$. 
Copyright 2020 BMJ Publishing Group. All rights reserved. For permission to reuse any of this content visit https://www.bmj.com/company/products-services/rights-and-licensing/permissions/

BMJ Case Report Fellows may re-use this article for personal use and teaching without any further permission.

Become a Fellow of BMJ Case Reports today and you can:

- Submit as many cases as you like

- Enjoy fast sympathetic peer review and rapid publication of accepted articles

Access all the published articles

Re-use any of the published material for personal use and teaching without further permission

Customer Service

If you have any further queries about your subscription, please contact our customer services team on +44 (0) 2071111105 or via email at support@bmj.com.

Visit casereports.bmj.com for more articles like this and to become a Fellow 